\title{
Kurze Mittheilungen aus der Entwicklungsgeschichte der Unke.
}

Von

Dr. Alexander Goette.

Ueber die Entwicklung des Eierstockseies, die Vorgänge bei der Dottertheilung und die Bildung der Keimblätter habe ich schon in früheren Mittheilungen das Wichtigste bemerkt. Hier setze ich dieselben fort und beginne daher mit der Bildung der Embryonalanlagen in den einzelnen Keimblättern.

Das obere Keimblatt. Es besteht aus zwei Zellenschichten, einer festeren hautartigen oberen-und einer locker gefügten tieferen. "Die letztere führt die Umbildungen des Keimblattes wesentlich herbei, die andere schliesst sich denselben meist nachträglich und ohne morphologische Veränderungen an; daher nenne ich jene die active, diese die passive Schicht des oberen Keimblattes. Die erste Umbildung der anfangs ziemlich gleichmässigen activen Schicht besteht darin, dass ihre Zellen von der Bauchseite theilweise auswandern, um sich an der Rückenseite in der Axenplatte anzusammeln. Diese Platte ist anfangs oval und ihre Verdickung ragt nach innen vor, woselbst sie im mittleren Theile ihrer Länge durch den Axenstreif, eine mediane, aufwärts vorragende Leiste des mittleren Keimblattes eingedrückt erscheint. Auf diese Weise erhält das Mittelstilck der Axenplatte zwei seitliche Bäuche, die Medullarplatten, welche durch ein dünneres Verbindungsstïck zusammenhängen; im Kopftheile wird diese Scheidung ebenso wie in der Schwanzgegend unkenntlich, da der Axenstreif in diesen Regionen verstreicht. Die ovale Form der Axenplatte geht alsbald in eine birnförmige über, indem ihre hintere Hälfte sich verschmälert, 
in gleichem Maasse aber auch länger wird. Die vordere Hälfte oder der Kopftheil bleibt einstweilen unverändert und tritt dadurch gegenüber dem beständig schmäler und länger werdenden Rumpftheile als eine breite mehr als halbkreisförmige Platte hervor.

Die Zellenansammlung zur Bildung der Axenplatte findet ihren Abschluss, sobald die übrige active Schicht auf eine einzige Zellenlage reducirt ist. Ausserhalb der Axenplatte bildet sie die Linse des Auges und die drei Seitennerven, um darauf mit den anliegenden peripherischen Theilen der passiven Schicht, welche für sich allein wieder die drüsigen Haftorgane erzeugt, zur Oberhaut zu verschmelzen, deren spätere Erzengnisse (Drüsen) daher nach jenen zwei ursprünglichen Schichten nicht mehr gesondert werden können.

Der Kopftheil der Axenplatte wird durch eine von unten eindringende Spalte in einen centralen runden, und einen denselben hufeisenförmig umkreisenden peripherischen Abschnitt geschieden die Hirn- und die Sinnesplatte. An der hinteren Grenze des Kopftheils gehen beide ungetrennt in die Medullarplatten über, deren lateraler Saum (später der hintere Rückenmarkstrang) also der Sinnesplatte entspricht. Die letztere hat verschiedene Schicksale in der hinteren und der vorderen Hälfte dès Kopftheils. Dort bleibt sie von der Hirnplatte gesondert und zieht sich zur Anlage des $\mathrm{Ohrbläschens} \mathrm{zusammen,} \mathrm{welches} \mathrm{bekanntlich} \mathrm{nur} \mathrm{von} \mathrm{der}$ aktiven Schicht gebildet wird. An den Seiten der vorderen Kopfhälfte hört die Scheidung der Hirn- und Sinnesplatte alsbald wieder auf, indem die Ietztere in die Hirnplatte ganz aufgenommen wird, dort den Seitentheil desselben bildet, welcher zur Bildung der A u genblase verwandt wird. Der unmittelbare Uebergang dieses Abschnittes der Sinnesplatte in die Anlage der Sehnerven und der Netzhaut lässt sich arn Batrachierembryo allerdings nicht nachweisen; ganz unzweifelhaft erscheint es aber an Embryonen der Knochenfische, an denen ich die Einsenkung zwischen beiden Platten in die Abschnürung der Augenblase übergehen sehe. - Am vorderen Umfange der Axenplatte bleibt jene Scheidung wiederum bestehen: seitlich entstehen aus der Sinnesplatte die beiden Geruchsplatten, das Mittelstück geräth durch die Vorwölbung des Vorderhirns unter dieses und wächst dann zwischen diesem und dem anliegenden Epithel der hinteren Mundhöhle erst trichterförmig, dann in Gestalt eines scheinbar soliden Zapfens nach hinten aus. Das. Ende desselben bleibt entweder oder wird doch sehr bald hohl und 
verwandelt sich, während die übrige Anlage, der Siel des angeschwollenen Endes verkümmert, in den Hirnanhang. Diese Entwickelung des Hirnanhanges ist aber nicht etwa auf die Batrachier beschränkt: an Säugethierembryonen finde ich es ganz ebenso, und beruht der Irrthum der bisherigen Auffassung einfach darin, dass man die frühzeitig schwindende Scheidewand zwischen der äusseren Mundbucht und der inneren Mundhöhle vor den Ausgangspunkt der Ausstülpungsbildung verlegte und diese erst in der geöffneten primitiven Mundhöhle entstehen liess. Ich sehe aber ihre Entwickelung vor der noch bestehenden Scheidewand, in dem Winkel zwischen dieser und der weit vorragenden Vorderhirngegend und zwar ganz offenbar vom oberen Keimblatte ausgehen.

Die Umbildung der Hirnplatte $\mathrm{zu}$ einem blasig geschlossenen Gebilde, dem Hirne, übergehe ich hier und will nur Einzelnes aus seiner späteren Entwicklungsgeschichte anführen. Für seine Morphologie sind namentlich 2 wei Punkte zu beachten: das Verhalten seiner Axe und seine ursprüngliche Eintheilung. Die Anlage des Hirns erscheint sehr frühe über das Vorderende der bleibenden Wirbelsaite etwa rechtwinklig nach unten abgebogen. Diese Knickung der Hirnaxe wird aber nicht, wie man bisher annahm, während der späteren Entwicklung wieder gehoben, sondern erhält sich vollständig auch im ausgebildeten Zustande des Hirns und wird nur durch das verschiedene Wachsthum der einzelnen Hirntheile verdeckt, wie sich aus dem Folgenden ergeben wird. - Die einzige ursprüngliche Eintheilung der Hirnanlage ist durch die Knickung gegeben, indem an dieser Stelle schon frühzeitig eine Einschnürung erscheint, welche eine vordere und eine hintere Hirnhälfte scheidet. Jene sondert sich erst verhältnissmässig spät in den vorderen unteren Theil (Vorderhirn), welcher unter dem Niveau der hinteren Hirnhälfte (Hinterhirn) liegt, und dessen Axe senkrecht verläuft, und in das keilförmige Verbindungsstück zwischen jenen beiden Hirntheilen, das Mittelhirn, dessen anfangs ganz kurzer Hohlraum den Uebergang vom horizontalen zum senkrechten Verlaufe der Hirnaxe enthält.

Das ursprüngliche Vorderhirn umfasst die Gegend der dritten Hirnkammer mit ihrer abwärts gerichteten Fortsetzung bis zur Sehnervenplatte; seine ursprüngliche Basis (Hinterwand) weitet sich später zum Hirntrichter aus, während seine Decke (Vorderwand) zu den beiden hohlen Grosshirnlappen auswächst, welche also nicht etwa aus einer medianen Einschnürung des ursprünglichen Vorder- 
hirns hervorgehen. Wenn also die Sehnervenplatte, die Mitte der anatomischen Hirnbasis, ursprünglich der Schlussseite des Vorderhirns angehört und gleichsam das Ende der Hirnaxe enthält, so wird der Weg von ihr zum oberen Theile der dritten Hirnkammer und von dort in die ursprüngliche Mittelhirnhöhle (den unteren Theil der späteren Höhle oder den aquaeductus Sylvii) der ursprünglichen Hirnaxe entsprechen, also stets wie diese aus dem horizontelen Verlaufe (aquaeductus S.) rechtwinklig nach unten gegen die Sehnervenplatte abgebogen erscheinen. Sowie die Grosshirnlappen hervorzuwachsen beginnen, verbinden sie sich mit den Geruchsplatten, indem einige zwischengeschobene Bildungszellen des mittleren Keimblattes eine Brücke zwischen beiden Organen herstellen; wo dieselbe mit dem Hirne zusammenhängt, entwickelt sich eine kleine Vorragung, der Riechnervenhügel, welcher unter dem hohlen Theile der Grosshirnlappen liegen bleibt. Indem nämlich aus der Vorderwand der letzteren solide und massige Fortsätze (lobi olfactorii ant.) hervorwachsen, rückt jener cerebrale Ursprung der Riechnerven nicht mit vor, sondern es zieht sich von dem Riechnervenhügel ein Strang aus, welcher dem soliden Hirnauswuchse bis zu dessen Vorderende eng angeschlossen bleibt und dort erst sich von demselben ablöst, um in den freien Riechnerven überzugehen. Dieser entspringt also nur scheinbar aus jenem Hirnauswuchse, und wenn sich ihm in späterer Zeit allerdings einige Nervenfäden aus dem letzteren hinzugesellen, so bleibt doch die eigentliche Ursprungsstelle immer der Riechnervenhügel. Der Ausdruck lobus olfactorius passt also für den bezeichneten Hirntheil nicht; dagegen ergiebt sich seine eigentliche Bedeutung aus dem späteren Verhalten. Die beiderseitigen Auswüchse verschmelzen nämlich zu einem Körper und denkt man sich die hohlen Abschnitte der Grosshirnlappen wie bei höheren Wirbelthieren nach allen Seiten, also auch über jene Verbindung hinaus erweitert, so springt die Homologie der letzteren mit dem vorderen Theile des Hirnbalkens in die Augen. - Der mittlere Theil der Decke oder des Gewölbes vom Vorderhirne wird in sehr früher Zeit ausserordentlich dünn, stülpt sich taschenförmig in die dritte Hirnkammer ein und verwandelt sich endlich, indem die Tasche von den äussern Hirnhüllen mit gefässreichem Bindegewebe gefüllt wird, in den epithelialen Ueberzug des daraus hervorgehenden Adergeflechtes. Dieses Epithel löst sich darauf aus dem Zusammenhange mit dem übrigen Hirne, wodurch das 
Gewölbe des Vorderhirnes eine wirkliche Lücke erhält, durch welche das Adergeflecht von Anfang an in die dritte Aderkammer hineinhängt. Hinter der Lücke entwickelt sich in dem genannten Gewölbe die Zirbel. Thre erste Anlage besteht in einer Substanzbrücke, welche beim Schlusse des Hirnes als letzter Rest von seinem Zusammenhange mit der Oberhaut zurückbleibt. Indem die Hirnhöhle sich in jene Substanzbrücke hineinzieht und dieselbe sich allmählich von der Oberhaut abschnürt, erscheint die Anlage der Zirbel als kleiner hohler Hirnauswuchs, welcher aber mit der Oberhaut stets in Berührung bleibt. Darauf sondert sich dieses Gebilde in einen bläschenartigen, der Haut anliegenden Theil und einen den letzteren mit dem Hirne verbindenden Stiel, welcher beständig länger und dünner wird und seine ursprüngliche Höhle verliert. Jene innige Anlagerung des Zirbelkörpers an die Oberhaut, welche niemals aufhört, lässt es natürlich erscheinen, dass derselbe bei der Bildung des Schädels ausserhalb des letzteren zu liegen kommt und bei jeder Abstreifung der Kopfhaut mit dieser entfernt wird. Vergleicht man nun die bisherigen Beschreibungen der Zirbel der Batrachier, so wird man sich leicht überzeugen, dass das wirkliche Organ bisher noch gar nicht gesehen oder erkannt worden ist, dass vielmehr das Adergeflecht damit verwechselt wurde. Die wirkliche Zirbel ist dagegen identisch mit der von Stieda beschriebenen „Stirndrüse des Frosches."

Am Hinterhirne finden sich weder ursprünglich, noch später Abtheilungen der ganzen Hirnröhre, welche Decke, Seitentheil und Basis zugleich umfassten, wie es beim Vorder- und Mittelhirn der Fall ist: Das verlängerte Mark entsteht vielmehr aus den vollständigen Seitentheilen und der Basis, also aus der ganzen untern Hälfte des Hinterhirnes; die obere Hälfte oder die Decke desselben zerfällt wiederum in einen kleinen vorderen Theil, welcher als Homologon des kleinen Hirnes bestehen bleibt, während der grössere hintere Theil sich vom Rande des verlängerten Marks und des kleinen Hirnes trennt und ähnlich wie es am Vorderhirne geschieht, sich in das Epithel eines Adergeflechtes verwandelt. Sucht man also nach gleichwerthigen Abschnitten des Hinterhirnes, so können als solche nicht das kleine Hirn und das verlängerte Mark, sondern nur das erstere und jenes Epithel des Adergeflechts gelten. Da nun alle diese Theile des Batrachierhirnes sich gerade so verhalten, wie das Hinterhirn an den Embryonen höherer Wirbelthiere, 
namentlich das in den Batrachiern vorhandene Homologon des kleinen Hirns mit der ersten Anlage desselben in höheren Abtheilungen übereinstimmt, so dürfte das von den Batrachiern Gesagte auch für die übrigen Wirthelthiere Geltung finden.

Ueber die Bildung der Netzhaut und des Sehnerven bemerke ich Folgendes. Die Anlage des letzteren oder der hohle Stiel der Augenblase (Netzhaut) mündet am unteren Umfange derselben. Die sogenanute Einstülpung der stets abgeplatteten Augenblase entstcht in der Weise, dass ihr Rand nach aussen verwächst, also mit dem zurückbleibenden Grunde einen doppelwandigen Napf bildet; indem dieses Wachsthum aber an der Einsenkungsstelle des Sehnerven ausbleibt, kommt derselbe an das Ende einer jenen Rand tief ausschneidenden Kerbe zu liegen. Die Bedeutung dieses Augenspaltes sehe ich darin, dass der Sehnerv unmittelbar nicht nur in die äussere Schicht der Netzhautanlage oder das Pigmentepithel übergeht, was beim Fehlen des Spaltes einträte, sondern auch mit einem Theile seiner Masse von Aufang an sich direkt in die innere nervöse Schicht einsenkt. - Die Lins e entwickelt sich als solide Wucherung der activen Schicht des oberen Keimblattes, welche erst uachträglich und ohne Vermittelung einer Einstülpung eine Höhle erhält. Indem die Aussenwand dieser Linsenblase sich verdünnt, die innere dagegen kugelförmig verdickt gegen die erstere vorragt, entsteht das Bild, welches die primäre Keimschicht mit der Dotterzellenmasse darstellt. Dieser Vergleich trifft um so mehr $\mathrm{zu}$, als jene Aussenwand als Linsenepithel die übrige kugelige, eigentliche Linsenmasse allmählich medianwärts umwächst. - Der Glaskörper und die Hornhaut (ausgenommen das Epithel) sind Producte des mittleren Keimblattes. - Vom Gehörorgan führe ich nur an, dass der Hörnerv sich gleichfalls aus dem mittleren Keimblatte entwickelt, vom Geruchsorga $n$, dass die ursprüngliche Geruchsplatte nur die mediale Wand der Nasengrube bildet, während die laterale aus dem Seitentheile des Gesichts hervorwächst.

Aus der Entwicklungsgeschichte der drei höheren Sinnesorgane ergibt sich, dass ihre homologen Anlagen nicht in drei Einstülpungen: Nasengrube, Linsen- und Ohrbläschen zu suchen, sondern in den drei gemeinsam aus der Sinnesplatte hervorgehenden Grundlagen der empfindenden Apparate, der Geruchsplatte, dem Augen-, Ohrbläschen enthalten sind. - Das Geschmacksorgan enthält keine Elemente des oberen Keimblattes, sondern entwickelt sich vollständig im Bereiche des Darmblattes. 
Schon während die Rü ckenmarksanlage sich in eine Röhre verwandelt, verwischt sich der Unterschied der activen und passiven Schicht in derselben. Darauf beginnt die Bildung der weissen Masse in der äusseren Zellenschicht des Rückenmarks. Dazu werden nur die äusseren Hälften der betreffenden Zellen verbraucht, indem sie in der Längsrichtung verschmelzend sich erst in eine durchsichtig homogene Masse verwandeln, in welchen später feinste Fasern erscheinen, von denen also eine grosse Anzahl auf den Querschnitt einer Zelle kommt. Die zugehörigen Zellenwände, so weit sie nicht in der Querrichtung schwanden, gehen an der Oberfläche des Rückenmarks in ein feines Häutchen, innerhalb der weissen Masse in die radialen von dem letzteren ausgehenden Scheidewände über. Die Zellen, welche die gráue Masse erzeugen, verschmelzen mit einander, wobei ein Theil der Kerne mit der sie zunächst umgebenden Masse Nervenzellen bildet. Die übrigen Kerne und Zellen gehn in die Bildung der zwischenliegenden. Nervenfasern und der bindegewebigen Zwischensubstanz ein. - Da die ursprüngliche Rückenmarksrinne bis in die Rusconi'sche Oeffnung hineinreicht, so setzt sich die daraus hervorgehende Röhre in den Darmkanal fort.

Das mittlere Keimblatt im Rumpftheile. Der schon genannte Axenstreif desselben entsteht wie die Axenplatte des oberen Keimblattes aus einer Zellenansammlung von den Seiten und rom Bauche her und zerfält in die Anlage der Wirbelsaite und die Segmentplatten, welche mit den sich an sie anschliessenden peripherischen Theilen des mittleren Keimblattes oder den Seitenplatten zweischichtig sind.

Die Anlage der Wirbelsaite wird sehr bald zu einem runden Strange, in dessen Zellen darauf durchsichtige Lakunen entstehen. Diese verdrängen allmählich die Zellsubstanz, so dass deren Reste mit den erhalten bleibenden Kernen schliesslich nur die Zwischenwände der Lakunen darstellen. Da aber innerhalb einer Zelle ebensowohl mehr als eine Lakune entstehen kann, als andererseits einige Lakunen unter einander zusammenfliessen, so gibt es solche mit mehren oder ohne Kerne; und da zudem die Zwischenwände einfach sind, so kann ich die bekannten grossen Fächer der Wirbelsaite nicht für Zellen halten. Alle ursprünglichen Zellen der Wirbelsaite erfahren dieselbe Umbildung; davon bleibt auch an der Oberfläche der Wirbelsaite keine intakte Zellenschicht zurïck. Aus den dort befindlichen Zellenresten entwickelt sich aber die innere Chorda- 
scheide. Die weiteren Schicksale der Wirbelsaite werden weiter unten angeführt.

Die Segmentplatten zerfallen in die Segmente, welche auch nach ihrer Trennung von den Seitenplatten zweischichtig bleiben innere und äussere Segmentschicht. Während die letztere жu jeder Zeit aus einer einfachen Zellenlage besteht, zerfällt die innere Segmentschicht in ihrem grösseren oberen Theile in eine mediale Platte, welche dem Rückenmarke und der Wirbelsaite anliegt - das innere Segmentblatt, und die Muskelplatte, welche zwischen dem letzteren und der äusseren Segmentschicht liegt. Die Zellen dieser Muskelplatte verwandeln sich in je eine Muskelfaser; die einseitig medianwärts und oberflächlich angelegte Muskelsubstanz jeder Zelle verdrängt erst allmählich deren übrige Substanz und den Kern, welcher bis zum vollständigen Schwunde der Dottersubstanz ungetheilt aber verlängert der Muskelsubstanz anliegt. Während er sich darauf vielfach theilt, spaltet sich auch die Muskelsubstanz der Länge nach erst in einige, dann in immer zahlreichere Säulchen, zwischen welche die Kerne sich einschieben und so ins Innere der Muskelfaser gelangen. Die Cohnheim'schen Felder halte ich für den Querschnitt jener Säulchen. - In jedem innern Segmentblatte bildet sich zunächst eine spindelförmige, nach unten strangförmig verlängerte Zellenmasse, im verdickten oberen Theile die Anlage des Spinalganglion!s, im unteren Strange des Spinalnervenstammes. Das Ganglion entwickelt sich ähnlich wie die graue Rückenmarkssubstanz. Die grossen rundlichen Nervenzellen haben anfangs keine Fortsätze; darauf lagern sich aber mit Ausläufern versehene Kerne an die Zellen, verschmelzen mit ihrer Substanz und bilden so deren Fortsätze. Die Ganglien verbinden sich je mit dem oberen Ende und der unteren Hälfte mit dem Rückenmarke. Diese Verbindungen ziehen sich:strangförmig aus und stellen so die zwei Nervenwurzeln vor, welche anfangs beide ins Ganglion münden. Erst später spaltet sich die untere (vordere) Wurzel bis zum gemeinsamen Stamme vom Ganglion ab, welches somit der oberen (hinteren) Wurzel allein verbleibt. Ebenso ist der Rückenzweig jedes Spinalnerven ein Auswuchs des Ganglions, welcher sich erst nachträglich von demselben abspaltet. - Die peripherischen Nerven entstehen aus Zellensträngen, welche in den Stämmen im Durchmesser mehrere Zellen enthalten, in den weiteren $Z$ weigen aber aus einzeln mit einander zusammenhängenden Zellen hervorgehen. Aus der Verschmelzung dieser Zellen entwickelt sich wie in der 
weissen Rückenmarksmasse eine feinfaserige mit Kernen besetzte Masse, und wenn, wie es wahrscheinlich ist, jede Faser einen Axencylinder oder eine marklose Nervenfaser bildet, so erhellt, dass jede Zelle nicht unmittelbar in eine Nervenfaser sich umbildet, sondern nach dem Verluste des eigenen Bestandes sich an der Erzeugung vieler Fasern betheiligt. - Die Marksubstanz entsteht aus kürzeren oder längeren Reihen von hellen rundlichen Körperchen, welche zwischen den Fasern iuftreten und allmählich mit einander verschmelzen. Wie sie sich zur Scheide eines Axencylinders umbilden, konnte ich nicht ermitteln.

Die ïbrige Masse des inneren Segmentblattes liefert das, ,interstitielle Bildungsgewebe" in der Umgebung der bereits genannten dorsalen Anlagen. Aus diesem Gewebe sondert sich frühzeitig eine Zellenschicht, welche sich der Wirbelsaite anlagert und über deren innerer Scheide eine solche äussere bildet. Die Zellen dieser äusseren Chordascheide verschmelzen zu einer homogenen Grundsubstanz, in welcher die vermehrten und abgeplatteten Kerne liegen, um welche sich später an bestimmten Stellen die Leiber von Knorpelzellen absondern, so dass mit der Bildung der letzteren auch zugleich eine Zwischensubstanz gegeben ist. So entsteht der byaline Knorpel überall. Die Knorpelbildung der äusseren Chordascheide vollzieht sich in deren oberer Hälfte, welche sich ansehnlich verdickt, namentlich entsprechend der Mitte jeder Muskelplatte, wo sie die Wirbelsaite durch quere Wülste tief einschnürt (Intervertebral-Knorpel). Die untere Hälfte der äusseren Chordascheide wird bindegewebig und liefert später Periost und Bandmasse. Während dieser Entwickelung der Wirbelkörper schrumpft die Wirbelsaite und plattet sich unter der genannten Knorpelmasse $a b$, wobei sie natürlich durch die röhrenförmige äussere Chordascheide stets in die embryonalen Wirbelkörper, wenn auch nicht in deren Knorpelmasse eingeschlossen bleibt. Auch geht sie durchaus nicht vollends zu Grunde, sondern indem sich ihr Fachwerk auflöst, bilden sich um die freien Kerne Knorpelzellen, deren ganze Masse sich alsdann dem übrigen Wirbelkörper anschliesst.

Ebenso wie die äussere Chordascheide sondern sich aus dem bezeichneten interstitiellen Bildungsgewebe an den Grenzen der Muskelplatten die Anlagen der Wirbelbögen ab. Diese entwickeln sich aber nicht gleich in ihrer ganzen Ausdehnung aus einer kontinuirlichen skeletogenen Schicht, sondern aus ganz kleinen, jener Chordascheide aufsitzenden Zellenhaufen und wachsen erst allmählich $\mathrm{zu}$ 
Knorpelspangen aus. Die beiderseits korrespondirenden verwacbsen aber nicht einfach in senkrechten queren Ebenen zu Ringen, sondern jede Spange bat einen senkrechten und einen sich daran rückwärts anschliessenden horizontalen Abschnitt, worauf sie sich erst medianwärts wendet, um über dem Rückenmarke mit der anderseitigen sich zu verbinden. Mit den dadurch entstandenen Ecken berühren sich die benachbarten Wirbelbögen von Anfang an und entwickeln daselbst ihre Gelenkfortsätze. Das früh verknöchernde Perichondrium zieht sich von der äusseren und der inneren Fläche jedes Wirbelbogens auf den folgenden hinüber; diese zweiblättrige Schicht verknöchert auch innerhalb des Rahmens, den jeder Wirbelbogen durch seine Biegungen über dem Rückenmarke bildet, verleiht ihm dadurch die spätere Breite seines oberen Theiles und gleicht seinen ursprünglich gebogenen Verlauf aus. - - Aus dem aufsteigenden Theile jedes Wirbelbogens entspringt eine lateralwärts gerichtete Knorpelspange, welche in die Muskelplatte hineinwächst und sie auf diese Weise in eine ohere und eine untere Masse theilt. Das Wurzelstück dieser Spange wird zum Querfortsatze des Wirbels; ihr lateraler Abschnitt sondert sich durch ein unvollkommenes Gelenk von ihm $a b$ und stellt mit seinem verknöcherten Körper und dem knorpelig bleibenden freien Ende eine Rippe dar. Doch verschmelzen diese Rippen einige Zeit nach der Larvenmetamorphose mit ihren Querfortsätzen, so dass nur an einzelnen Wirbeln sich die Spuren der frühercn Trennung erhalten.

Hinter dem neunten selbstständigen Wirbel zeigen sich noch die Anlagen von vier weiteren Wirbeln, welche jedoch so vollständig zu einer Röhre verwacbsen, dass jederseits nur noch zwei ZwischenWirbellöcher die früheren Grenzen des 10.-12. Wirbels andeuten. Die Bildung dieses hintersten Abschnittes der Wirbelsäule weicht aber insofern $a b$, als auch an der Bauchseite der Wirbelsaite sich ein Knorpelstreif bildet, so dass die letztere allseitig ron Knorpel und Knochen umschlossen wird. Ihr im Larvenschwanze befindliches freies Ende wird während der Verkümmerung desselben unter Bildung von zahlreichen Querfalten zusammengeschoben, ehe es ganz verloren geht.

Um die Entwickelung der übrigen Erzeugnisse des interstitiellen Bildungsgewebes, welches übrigens nicht nur aus dem inneren Segmentblatte, sondern auch an anderen Stellen des mittleren Keimblattes entsteht, $\mathrm{zu}$ verstehen, muss man seine eigene ursprüngliche Bildung kennen. Seine erste Anlage besteht aus einer 
Zellenmasse, deren indifferente Elemente sich allseitig berühren. Mit einer Vergrösserung des für diese Anlage bestimmten Raumes ergiesst sich eine Flüssigkeit zwischen die Embryonalzellen und drängt sie auseinander; dabei ziehen sich die mehrfachen zwischen ihnen bereits entstandenen Verbindungen zuerst in Form kurzer Brücken, dann fadenförmig aus. So besteht das genannte Gewebe alsbald aus einem Netzwerke zusammenhängender Zellen, dessen Zwischensubstanz von aussen herzukam. Dieses Netzwerk nimmt zu und breitet sich aus durch die einwandernden embry on a len Blutoder Dotterbildungszell e n, welche hier und dort hängen bleiben, Fortsätze ausziehen und bei der stetigen Wachsthumsverschiebung des Ganzen neue Verbindungen finden, um endlich vollständig in dasselbe sich einzufügen. Diese Zellen, welche als erste embryonale Wanderzellen allen Geweben neue Elemente zuführen, stammen aus den Anlagen der $\mathrm{Ha}$ up tge fäs se, welche als Intercellulargänge aus dem Bildungsgewebe hervorgegangen, anfangs netzförmig durchbrochene Wände haben, so dass die Blutzellen leicht hinaustreten können. Anders wie die grossen Gefässstämme entwickeln sich die kleinen Gefässe: indem der Inhalt einzelner Zellen des Bildungsgewebes sich verflüssigt und diese kleinen mit Flüssigkeit gefüllten Räume durch die überall vorhandene Zellenverbindung mit einander zusammenfliessen, entstehen kürzere oder längere Intracellularräume, welche mit den schon vorhandenen Gefässen anfangs nur durch das allgemeine Zellennetzwerk zusammenhängen und erst nachträglich durch fortgresetzte Kanalisation der zwischenliegenden Theile zusammentliessen. Später geht die Bildung neuer Gefässe allerdings von den schon bestehenden aus, aber ebenfalls nur durch die Aushöhlung jenes Zellennetzes, welche in diesem Falle vom drängenden Blutstrome ausgeht. Die bisherige Lehre von der Gefässbildung kann ich nicht bestätigen. - Aus der speziellen Entwicklungsgeschichte des Gefässsystems hebe ich nur die Thatsache hervor, dass die hintere Hohlvene mit ihrer vorderen Hälfte im Lebergekröse entsteht, am grösseren hinteren Abschnitte aus den Kardinalvenen hervorgeht.

Das fibrilläre Bindegewe be entsteht in der Weise, dass einige Zellenstränge des Netzwerkes zu stärkeren Balken oder Blättern verschmelzen, in denen die einzelnen Zellen untergehen. Aus der Masse der Zellenleiber gehen die Bindegewebsfibrillen hervor, denen die freien Kerne als die eigentlichen Bindegewebskörperchen angefügt bleiben. Alle wirklich zelligen Elemente des Binde- 
gewebes sind nachträglich eingewanderte, welche in den Bestand des genannten Gewebes nicht eingehen, also auch eigentlich nicht zu demselben gehören. Die Zwischenräume der Bindegewebsmassen werden zu Ly mphräumen, wohl zu unterscheiden von den intracellulären Lymphgefässen, welche genau so wie die secundären Blutgefässe entstehen, so dass auch das lymphatische Gefässsystem zwei dem Blutgefässsystem entsprechende Entwicklungstypen hat.

Wenn die schon beschriebenen Muskelplatten nur für die Wirbelsäule bestimmt sind, so entwickeln sich die übrigen Mnskeln der Leibeswand nebst den Gliedmassen und allem zugehörigen interstitiellen Bildungsgewebe dennoch gleichfalls aus den Segmenten und zwar in den auswachsenden Segmentschichten. Die bisher betrachteten Körpertheile entstanden aus der grösseren oberen Hälfte des inneren Segments. Die untere Fülfte wächst mit der äusseren die dorsale Muskelplatte deckenden Segmentschicht zwischen der Oberhaut und den Seitenplatten hautartig abwärts, so dass die beiderseitigen inneren und äusseren Segmentschichten am Bauche zusammentreffen. Die in nere Segmentschicht behält die segmentale Eintheilung und schiebt sich mit ihrem vorderen Ende an der Bauchseite des Kopfes, welcher eine solche Embryonalanlage nicht besitzt, bis zum Unterkiefer vor. Hinten umgreift sie den Afterdarm, um dicht über ihm zu endigen. In dieser Schicht entsteht in ganz dünner Lage der mittl ere Bau chm uskel, welcher jederseits vom Rücken bis zur Bauchseite abwärts reicht. Die Entwicklung seiner Fasern unterscheidet sich aber nicht "unwesentlich von der schon beschriebenen, in den dorsalen Muskelplatten beobachteten. Jede Faser entsteht nämlich nicht aus je einer, sondern aus mehreren mit einander verschmelzenden Zellen; und ebenso verhält es sich in allen übrigen noch zu erwähnenden Muskeln. Während der Larvenzeit schiebt sich der mittlere Bauchmuskel abwärts zusammen und sondert sich in die Mm. genio- und sternohyoidei, rectus abdomnis und perinealis. Zwischen den $\mathrm{m}$. sternohyoideus und rectus abdominis bildet sich jederseits ein bauchrippenähnliches Skeletstück; beide verschmelzen nach vorn convergirend in der Bauchlinie und bilden die Schenkel des als Hyposternum der Unke bekannten Skeletstückes. Bei den Tritonen geht das Mittelstück des genannten Bauchmuskels nicht vollständig in einen $m$. rectus abdominis auf, sondern zerfällt in einen solchen und einen m. obliquus internus. Die äussere Segmentschicht erzeugt die beiderlei Gliedmassen, ausser- 
dem zwischen ihnen den $\mathrm{m}$. obliquus externus und zwischen Kopf und Schultergürtel noch den $\mathrm{m}$. sternocleidomastoideus, welcher von jenem Gürtel getrennt entsteht, wogegen der m. omohyoideus zu demselben gehört. Die breite, mediane Platte des Hyposternum bildet sich getrennt von dem Bauchrippenstück und verbindet sich erst nachträglich mit demselben. - Der m. quadratus Iumborum gehört dem Beckengürtel an und rückt erst nachträglich in seine bleibende Lage vor. - Ausser den genannten Muskeln gibt es noch einen inneren Ba uch muskel (m. transversus abd.), welcher aber aus keiner morphologischen Embryonalanlage, sondern verhältnissmässig spät unmittelbar auf dem parietalen Bauchfelle und neu eingeführtem Bildungsgewebe hervorgeht.

Die Seitenplatten wachsen mit ihren medialen Rändern zwischen den Segmenten und dem Darmblatte gegen die Medianebene, wo sie sich zwischen der Wirbelsäule und dem Darme vereinigen. Von ihren beiden Schichten, die alsbald auseinandertreten, um die Bauchhöhle zu bilden, verwandelt sich das äussere, der Leibeswand anhaftende Parietalblatt in den parietalen Bauchfelüberzug, und das dem Darmblatte anliegende Visceralblatt in das ganze viscerale Bauchfell und darunter in die bindegewebigen und muskulösen Theile des Darms und seiner Anhangsorgane. Durch Ausstülpung aus dem Parietalblatte entsteht ferner die Urniere mit ihrem Ausführungsgange; sie besteht aus dem aufgeknäuelten Ende des Ganges, welcher zuletzt dreiarmig in die Bauchhöhle mündet. Gerade gegenüber dieser Mründung liegt der Gefässknäuel, welcher aus dem Visceralblatte hervorgeht. Die bleibende Niere entwickelt sich gleichfalls aus der Seitenplatte, indem deren mediale Falte zwischen dem Urnierengange und der Aorta in den Retroperitonealraum hineinwächst, sich darauf abschnürt und in eine Reihe getrennter kleiner Schläuche zerfällt. Diese wachsen zu den Harncanälchen aus, ihre soliden kolbigen Enden verwandeln sich in Gefässknäuel. Medianwärts von den Nieren entwickelt sich die leistenförmige Anlage der Geschlechtsorg a ne aus dem Parietalblatte; anfangs sind dieselben für beide Geschlechter gleich, aus Follikeln zusammengesetzt: die in der eingeschlagenen Richtung fortlaufende Entwicklung derselben liefert den Eierstock, ein gewisser Stillstand und Rückgang in derselben die Hoden. Der Hermaphroditismus der gemeinen Kröte beruht darauf, dass das in der Entwicklung voraneilende Kopfende des indifferenten Geschlerhts- 
organes, sowie es dem jungen Eierstocke die grössten Eifollikel liefert, bei dem Eintritte der Hodenentwicklung bereits $z u$ weit vorgeschrittene Follikel enthält, um dieselben noch in Hodenschläuche verwandeln zu können.

Das mittlere Keimblatt im Kopfe. Im Kopftheile erscheint die Anlage der Wirbelsaite anfangs bis unter das Vorderhirn fortgesetzt; doch schwindet dieser ihr vorderster Abschnitt sehr bald. Die Segmentplatten zerfallen jederseits in vier Segmente, von denen das erste Paar der vorderen Stirnhälfte anliegt, die drei übrigen die Seiten des Hinterhirnes einnehmen, eines vor, die beiden andern hinter dem Ohrbläschen. Die äussere Segmentschicht oder die vier äusseren Segmente überwiegen die inneren bedeutend und wachsen an der Seite hinab, während die letzteren auf die Rückenseite beschränkt bleiben. Die Seitenplatten verschwinden im Vorderkopfe ganz, im Hinterkopfe erhalten sie sich in der Schlundwand theilweise, vollständig in dem darunter befindlichen Herzraum. Die sehr verwickelte Umbildung des Kopfes aus jenen einfachen morphologischen Elementen im kurzen Auszuge darzustellen, ist unmöglich; daher beschränke ich mich hier darauf, die Erzeugnisse der einzelnen Segmente aufzuzählen, wobei deren Homologien bereits erkennbar werden.

Das erste innere Segment liefert die Augenmuskeln, deren Nerven mit den $n$. nasalis trigemini aus einer gemeinsamen Anlage hervorgehen ; ferner das erste Wirbelbogenpaar des Kopfes (R a th ke's seitliche Schädelbalken), welches zur Grundlage der vorderen Schädelkapsel wird. Aus der vorderen Vereinigung beider Bögen wächst die Nasenscheidewand, der Zwischen- und Oberkieferknorpel hervor. Das Schädeldach ist niemals vollständig knorpelig, so dass die später knorpelfreien Theile erst durch Resorption entständen, sondern die seitlich hervorwachsenden Knorpelbrücken sind vielmehr anfangs unter der Knochenplatte viel schmächtiger als später. Während der Larvenzeit besteht eine rudimentäre Anlage eines harten Gaumens und eines Nasenrachenganges, welche später schwindet. Das erste äussere Seg ment erzeugt die Kaumuskeln mit den beiden Kiefernerven, deren gemeinsames Ganglion mit demjenigen der Nasenund Augennerven zum Gasser'schen Nervenknoten verschmilzt. Ferner stammen noch von demselben Segmente die Lippenmuskel ab. Das zugehörige .Skeletstück umfasst znnächst einen Knorpelbogen, welcher von vorn nach hinten das Gaumen-, Flügel-, Quadratbein und den grossen Flügelknorpel (ala magna) enthält; endlich den 
früh abgesonderten Unterkiefer. - Die drei inneren Segmente des Hinterko $\mathrm{p}$ fes entwickeln Muskelplatten, welche als eine Fortsetzung derjenigen des Rumpfes erscheinen, aber noch während der Larvenzeit schwinden. Neben ihnen entsteht die hintere Schädelbasis, in deren continuirlicher Anlage die Wirbeleintheilung fehlt. Mit Rücksicht auf die Entwicklung des gleichfalls ungegliederten Schwanztheils der Wirbelsäule, wo auf jedes Segment die Anlage eines Wirbels kommt, muss man aber jene Schädelbasis als drei Wirbeln entsprechend ansehen; deren Bögen jedoch nur am letzten oder den beiden letzten zur ringförmigen Entwicklung gelangen. Zwischen diesem occipitalen Wirbelringe und der vorderen Schädelbasis (erstes Wirbelbogenpaar) wird die seitliche Schädelwand vom eingeschobenen grossen Flügelknorpel (erstes äusseres Segment) und der Ohrkapsel gebildet; die Knorpelränder und -Brücken des entsprechenden Schädeldaches entstehen theils aus einem Fortsatze der vorderen Schädelkapsel (ala parva), theils aus dem occipitalen Wirbelringe.

Das zweite äussere Segment liefert die Muskeln des Zungenbeinbogens und einen Nervenknoten, aus welchem wie am Unterkieferbogen ein sensibler und ein motorischer Nerv hervorgehen (n. palatinus und n. facialis). Das ganze Zungenbein ist Erzeugniss von Resten der Seitenplatte, welche zwischen dem Herzraum und dem Unterkiefer zurückblieben; es ist daber weder diesem letzteren noch dem Brustbein homolog, während der ganze Unterkieferbogen und die beiden Gliedergürtel durchaus gleichwerthige Theile sind. Aus dem dritten und vierten Segmente entstehen Kiemenmuskeln, welche später theilweise sich in Schlundmuskeln verwandeln, uud ferner zwei entsprechende Nervenstämme, der n. glossopharyngeus und der Stamm des $n$. vagus mit ihren Ganglien. Der letztere ist als einem einzigen Segmente angehörig, einfach angelegt, wird aber durch die Ausbildung des Kiemenapparates dreitheilig, für den zweiten bis vierten Kiemenbogen. Da der letzte Kiemenbogen gleichsam seine Bestimmung nicht erreicht, sondern sich in den Boden eines Vorhofs des Kehlkopfs verwandelt, so wird der dritte ursprüngliche Ast des n. vagus zum n. laryngeus anterior. Die übrigen Aeste des fertigen Vagus wachscn nicht aus ihm heraus, sondern sind selbstständige Nervenanlagen, welehe sich erst nachträglich ihm anschliessen; so die aus der Oberhaut hervorgehenden nn. laterales superior et inferior und der in dem Visceralblatte der Speiseröhre entwickelte Eingeweideast, der scheinbare 
Hauptstamm des n. vagus, dessen Entwickelung jedoch ihm eine von allen übrigen Nerven ganz verschiedene Stellung anweist. (Mit gleicher Selbstständigkeit entsteht der Sympathicus, dessen Verbindungen ebenso erst nachträglich sich vollziehen.)

Aus der Entwicklungsgeschichte der $\mathrm{Ki}$ emen will ich nur hervorheben, dass die Kiemenbögen noch vor der Ausbildung der äusseren Kiemen zu queren Platten auswachsen, so dass, sobald dieselben sich von einander entfernen, die früheren Spalten sich zu ansehnlichen Räumen erweitern, den inneren Kiemenhöhlen, welche sowohl gegen den Schlund einen gewissen Abschluss, als auch an ihren Wänden echte in n ere Kie mien (Rusconi's „filtre") besitzen Die bisher bekannten Kiemen sind nicht als äussere und innere, sondern nur als obere und untere A ussenkiemen zu unterscheiden.

Zum Kopfe gehörig muss noch das Herz mit der es. umgebenden Höhle betrachtet werden, da es aus der ventralen Seitenplatte des Kopfes entsteht und zwar im Allgemeinen so, wie ich schon vor Jahren darstelltc. Die Perikardialhöhle ist daher als eine Fortsetzung der Bauchhöhle anzusehen, welche aber von letzterer durch das Lumen versperrende Organe getrennt wird, in deren Umfange alsdann das Parietal- und Visceralblatt verschmelzen. Diese Organe sind an der Decke der Pericardialhöhle die Lungenwurzel, rückwärts die Leber.

Das D armblatt. Es umschliesst anfangs den embryonalen Darmraum vollständig nur im Vorderdarme; im Mittel- und Hinterdarme bildet es gewissermassen bloss ein Dach über dem Darmraume, dessen Ränder ohne Abgrenzung in die Dotterzellenmasse übergehen. Die letztere ist die Keimstätte des embryonalen Blutes, welches an ihrer Obertläche aus einer raschen Theilung einzelner Dotterzellen hervorgeht. Die zuerst isolirten Blutbildungsheerde fliessen netzförmig zusammen und dieses stets mit Blut gefüllte, rinnenartig in der Dotterzellenmasse ausgegrabene Gefässnetz breitet sich unter dem Visceralblatte so weit aus, bis es an die Leber und den darüber offenen, gleichfalls aus dem Visceralblatte hervorgegangenen Venensack gelangt, um dort das Herz zu füllen. Nachdem die Blutbildung ihr Ende erreicht hat, umwächst das Darmblatt den Rest der Dotterzellenmasse, weicher im Darmraume aufgelöst wird, denselben dadurch erweitert und zugleich die erste Nahrung bietet (Nahrungsdotter). Das Darmblatt geht ins Epithel des Darmes und seiner Anhangsorgane - Schilddrüse, Lunge, Leber, Bauchspeicheldrüse, Harnblase - über. Denn auch die 
embryonale Leber ist aus Drüsengängen zusammengesetzt; das bekannte Lebernetz besteht nicht aus soliden, sondern hohlen Sprossen, welche aber, wenigstens zum grössten Theile, nicht blind endigen, sondern sich unter einander verbinden. In den Zwischenräumen fliesst das Blut anfangs frei, die Leber ist wie ein Schwamm, welchen alles neue Dottervenenblut durchsetzen muss, um ins Herz zu gelangen. Da jene Bluträume von Anfang an nur wenige hintere Eingänge und vordere Ausmündungen besitzen, aber je tiefer ins Innere hinein, desto reicher mit der zunehmenden Verzweigung des Lebernetzes sich gleichfalls verzweigen, so sind ein zu- und ein abführendes Gefässsystem schon durch diese ursprüngliche Verhältnisse angelegt, ohne dass verschiedene Gefässe von mehreren Seiten hineinzuwachsen brauchten. - Die Bauchspeicheldrüse entsteht in ihrer Hauptmasse hinter der Leber mit einem Gange; eine kleinere Anlage sprosst rechts von dem Lebergange hervor und verbindet sich mit der ersteren. Während aber die beiden verhäItnissmässig weit auseinanderliegenden Ausführungsgänge vergehen, wird ein dritter links vom Lebergange entstehender und über denselben die Bauchspeicheldrüse erreichender zum definitiven ductus pancreaticus.

Noch habe ich eine Bildung zu erwähnen, welche erst in der späteren Larvenzeit, nachdem die Embryonalanlagen als solche verbraucht sind, aus indifferentem Bildungsgewebe entsteht; es ist die Milz. Ihre Anlage ist ein kleiner solider Zellenhaufen im Mesenterium. Sobald die Dotterzellenmasse geschwunden ist, also auch die Bildung des Dotterblutes aufgehört hat, entwickeln sicn in jener Anlage helle runde Zellen in zunehmender Anzahl, welche darauf in die Blutbahnen gespült zu den weissen Blutkörperchen werden, die den steten Ersatz des Blutes besorgen.

Die vorstehenden Mittheilungen stellen einen kurzen und zwar nur theilweisen Auszug aus einem gröseren Werke dar, welches bereits in Druck gegeben ist, dessen Erscheinen jedoch wegen seines. Umfanges vor der zweiten Hälfte des nächsten Jahres nicht zu erwarten ist. Ich glaubte daher einige Resultate meiner Untersuchungen, welche mir besonders beachtenswerth erscheinen, hier vorausschicken $\mathrm{zu}$ dürfen.

4. September 1872. 